
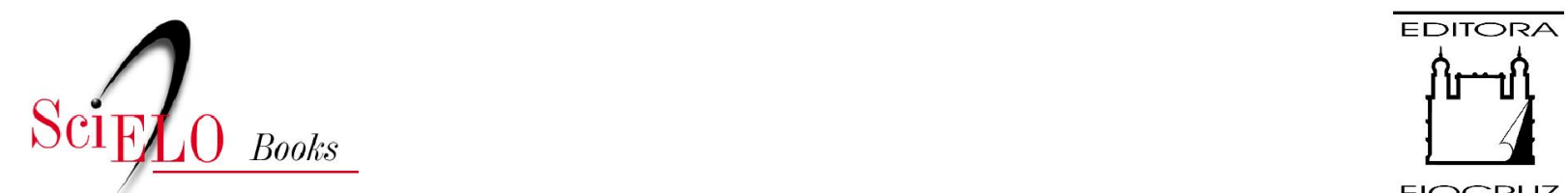

FIOCRUZ

\title{
El reto de la educación digital
}

más allá de la transformación metodológica

\section{Rosa Ana Martín Vegas}

\section{SciELO Books / SciELO Livros / SciELO Libros}

VEGAS, R. A. M. El reto de la educación digital: más allá de la transformación metodológica. In: SOUZA, F. M., and ARANHA, S. D. G., orgs. Interculturalidade, linguagens e formação de professores [online]. Campina Grande: EDUEPB, 2016, pp. 251-272. Ensino e aprendizagem collection, vol. 2. ISBN 978-85-7879-347-0. Available from: doi: $10.7476 / 9788578793470.0012$. Also available in ePUB from: http://books.scielo.org/id/qbsd6/epub/souza-9788578793470.epub.

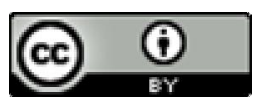

All the contents of this work, except where otherwise noted, is licensed under a Creative Commons Attribution $\underline{4.0 \text { International license. }}$

Todo o conteúdo deste trabalho, exceto quando houver ressalva, é publicado sob a licença Creative Commons Atribição 4.0.

Todo el contenido de esta obra, excepto donde se indique lo contrario, está bajo licencia de la licencia Creative Commons Reconocimento 4.0 . 


\section{EL RETO DE LA EDUCACIÓN DIGITAL: más allá de la transformación metodológica}

Rosa Ana Martín Vegas (USAL)

La educación digital se extiende de forma natural como consecuencia de la expansión de las tecnologías de la información y de la comunicación (TIC) y su impacto social. El debate sobre si la incorporación de las TIC a las aulas es una necesidad educativa o una cuestión de esnobismo está ya fuera de duda, pues claramente la escuela no puede estar de espaldas a la sociedad y no puede desaprovechar los flujos de información que parten de una fuente ingente, de máxima actualidad y de mayor accesibilidad para todos como es internet. En este artículo plantearemos los cambios requeridos para la eficacia de este nuevo modelo de metodología formativa a partir del análisis de las deficiencias en su implantación actual, heredadas, en gran medida, de la inercia de un modelo educativo tradicional y difícil de superar.

\section{¿Por qué es necesaria una revolución educativa?}

Se habla de altos índices de fracaso escolar, de desmotivación y desinterés por parte de los alumnos, de ineficacia del sistema y, en general, de un sistema educativo que 
no forma a ciudadanos preparados para el mundo laboral y la vida comunitaria real. Esta crítica profundamente arraigada en la educación española, en gran parte de la europea y en la del continente iberoamericano procede, por un lado, de la idea negativa muy extendida de que el sistema actual no funciona, y reclama, por otro lado, innovación; algo no funciona y hay que cambiarlo. En España, cada gobierno de turno ha realizado una reforma educativa siempre contando con la oposición de gran parte de la comunidad educativa. Finalmente, dichas reformas no se han llevado a cabo en su totalidad, bien porque el espíritu ideológico de la ley no ha llegado a la práctica del aula (la LOGSE de 1990, por ejemplo) o bien porque la plena implantación se ha obstruido con cambios políticos. Este deseo de cambios en el sistema educativo es un hecho derivado de que lo que hay no funciona bien y del deseo de innovar, que es una necesidad educativa de primer orden. Y es aquí de donde parte nuestra reflexión.

¿Tan malo es el sistema actual? ¿Es malo porque hay alumnos que no llegan a obtener la aptitud de los estudios obligatorios, porque los alumnos que certifican están muy mal preparados o por las dos cosas? ¿Por qué los alumnos están mal preparados? ¿Porque no saben nada, porque no saben hacer nada, porque lo que les han enseñado no les sirve para nada...? ¿Cuáles son las razones de que el sistema no funcione? ¿Los profesores, la actitud de los alumnos ante la escuela, la falta de prestigio social del colegio, los recursos que se utilizan para aprender o la falta de medios económicos en algunos casos? Podríamos continuar con más preguntas sobre qué es lo que pasa para justificar la necesidad de cambios, pero, probablemente, ni todo lo que hay es malo y debe liquidarse, ni se deba 
plantear la innovación como la erradicación de lo existente, sino como un proceso de mejora y de adaptación a la vida.

Son muchos los artículos escritos por distintas autoridades en el ámbito educativo que discuten el origen del mal y promulgan la revolución educativa. Centran su atención en aspectos como que la educación no tiene conexión con el mundo real, cuestión causante de la desmotivación del alumnado, que no tiene interés por aprender lo que no tiene una utilidad inmediata. Afirmaciones de este tipo carecen de absoluta veracidad, pues en la escuela se enseña a conocer el mundo en sus múltiples facetas imposibles de experimentar de forma individual. El problema de parte de este desinterés del alumnado no reside en los conocimientos que se enseñan sino en cómo se enseñan. $Y$ el cómo va mucho más allá de los recursos utilizados que discutiremos más adelante. Se trata de enseñar de manera transversal, relacionando contenidos, ideas, emociones, experiencias... para mostrar a los escolares la conectividad del mundo real con sus complejos nódulos estructurales. De esta forma, cada individuo podrá ver y disfrutar de la utilidad del conocimiento, porque un aprendizaje redundará en otro y este a su vez en otro que cada alumno podrá aplicar a sus propios intereses.

El DRAE define la palabra revolución como un "cambio rápido y profundo en cualquier cosa". La revolución educativa tal y como la vamos a concebir en este estudio no puede ser rápida, pues va más allá del cambio instrumental que supone la introducción de las TIC, pero sí es profunda, porque exige cambios en el planteamiento metodológico que requieren un profunda formación conceptual en el dominio de cada materia por parte del profesorado y una transformación en los hábitos de transmisión 
que permitan el feedback del alumno para que procese e interiorice su aprendizaje.

\section{Métodos revolucionarios de la escuela actual}

Esta supuesta revolución que hay que llevar a cabo en el sistema educativo se ha etiquetado con el término innovación. La acción de innovar, introducir novedades, debe existir en la enseñanza de cualquier materia siempre, cada día, cada curso... Trabajar con personas exige la continua adaptación a las capacidades intelectuales, perceptivas, físicas, sociales y personales de cada individuo. No es posible en educación seguir la misma pauta con alumnos pertenecientes a distintas generaciones, edades, niveles y capacidades. Por eso, siempre hay que innovar, siempre que buscar métodos de acercamiento a los distintos discentes y grupos para desarrollar sus facultades intelectuales y morales, así como para enseñarles los buenos usos de urbanidad y cortesía -como define la palabra educación el DRAE-, que deben contextualizarse en cada sociedad y acto de convivencia. Por tanto, innovación no es sinónimo de educación digital, pues se puede innovar usando los llamados recursos tradicionales. Innovación implica cambio metodológico, adaptación a las capacidades y necesidades del alumno con el fin de que logre el aprendizaje. El cambio metodológico es motivador, pues despierta la atención de los alumnos ante lo nuevo. Por eso, no siempre es innovación el uso de las TIC; se puede ser muy innovador utilizando recursos de muy distinta tipología.

La innovación educativa se ha centrado en los recursos metodológicos. Casanova y Bazarra (2016) señalan distintos métodos como revolucionarios en la escuela actual: 
1) el aprendizaje basado en proyectos: desarrollar un proyecto para dar solución a un problema real implicando a varias asignaturas; 2) la gamificación: usar mecánicas de juego para desarrollar habilidades; como en los videojuegos, hay retos, avatares, fases y niveles; 3 ) la flipped classroom (la clase al revés): las lecciones que se escuchaban en el aula ahora se oyen en casa mediante grabaciones y los deberes que tradicionalmente se hacen en casa, ahora se hacen en la clase mediante trabajo en equipo; 4) el design thinking: el alumno piensa como un diseñador y debe seguir los pasos de empatizar, definir el problema, idear, aplicar y evaluar; 5) el design for change: se llama aprendizaje servicio porque se trata de que los alumnos hagan un servicio a su comunidad para aprender a colaborar y desarrollar la conciencia social; por ejemplo, los alumnos del instituto de Sils (Gerona, España) acuden al geriátrico del municipio; 6) el aprendizaje cooperativo: trabajar en grupos pequeños y aprovechar las capacidades y conocimientos de cada miembro; y 7) el aprendizaje basado en problemas: los alumnos deben estudiar un problema para resolverlo y deben concurrir conocimientos de varias asignaturas.

¿Son realmente estos métodos revolucionarios? Muchos de ellos son antiquísimos y otros, aunque sin la misma denominación, se llevan a cabo en algunas aulas desde hace décadas. Sin embargo, sí son revolucionarios porque no son populares, es decir, no se aplican en la gran mayoría de las escuelas y responden a una manera de trabajar que no es la frecuente en los sistemas educativos de la mayor parte de los países. Al margen de la etiquetación de las estrategias metodológicas, comparten ciertos rasgos que rompen con la estructura tradicional de las clases (cuadro 1). 
Cuadro 1 - metodología educativa tradicional vs. metodología "innovadora" actual

\begin{tabular}{|l|l|}
\hline \multicolumn{1}{|c|}{ clase tradicional } & \multicolumn{1}{c|}{ clase "innovadora"1 } \\
\hline $\begin{array}{l}\text { clase magistral: el profesor } \\
\text { explica, los alumnos escuchan y } \\
\text { pueden tomar notas }\end{array}$ & $\begin{array}{l}\text { trabajo por proyectos (distintas } \\
\text { modalidades): el profesor pauta } \\
\text { una guía de trabajo y los alumnos } \\
\text { adquieren conocimientos y } \\
\text { desarrollan habilidades por sí mismos }\end{array}$ \\
\hline $\begin{array}{l}\text { los contenidos se definen por } \\
\text { materias }\end{array}$ & $\begin{array}{l}\text { los contenidos son transversales, } \\
\text { implican a varias áreas de } \\
\text { conocimiento y consienten una } \\
\text { comprensión global e integradora }\end{array}$ \\
\hline $\begin{array}{l}\text { trabajo individual: los alumnos } \\
\text { realizan ejercicios para procesar } \\
\text { toda la información recibida en la } \\
\text { clase magistral }\end{array}$ & $\begin{array}{l}\text { trabajo cooperativo y en grupo: los } \\
\text { alumnos exploran los contenidos, } \\
\text { los procesan y resuelven en } \\
\text { colaboración, compartiendo el } \\
\text { aprendizaje en el grupo }\end{array}$ \\
\hline $\begin{array}{l}\text { recursos: libros de texto y fichas } \\
\text { con ejercicios }\end{array}$ & $\begin{array}{l}\text { recursos: libros, revistas, } \\
\text { periódicos, blogs, bases de datos...; } \\
\text { materiales digitales en red }\end{array}$ \\
\hline $\begin{array}{l}\text { evaluación: a través de exámenes } \\
\text { y pruebas individuales se } \\
\text { comprueba si los alumnos han } \\
\text { asimilado los conocimientos } \\
\text { enseñados }\end{array}$ & $\begin{array}{l}\text { autoevaluación y coevaluación: } \\
\text { se evalúa no solo resultados, } \\
\text { también el proceso y el progreso } \\
\text { de aprendizaje con autocrítica } \\
\text { (individual y de grupo) para que } \\
\text { los escolares sean más conscientes } \\
\text { de su desarrollo }\end{array}$ \\
\hline
\end{tabular}

Fuente: Rosa Ana Martín Vegas.

Se habla de hasta veinte retos para la educación del futuro (PÉREZ-BARCO, 2013): formar al ciudadano del

1 Clase "innovadora", entrecomillado, porque realmente la enseñanza siempre debe ser innovadora, y la innovación, pese a utilizarse ahora como sinónimo de modernidad y de uso de recursos nuevos, ha existido siempre en cada época. 
siglo XXI, centrar la inclusión social como eje, buscar el liderazgo internacional, extraer la inteligencia colectiva, ligar conocimientos, pedagogía y tecnología, implicar nuevos métodos de evaluación con las TIC, romper el mito de los nativos digitales, ${ }^{2}$ fomentar la creatividad, valorar la educación emocional, conseguir la cooperación entre la familia, la escuela y la comunidad, centrar el liderazgo en la pedagogía y no en la burocracia, tener presente que el objetivo es siempre el desarrollo de competencias, focalizar la enseñanza en los intereses del aprendiz, reconfigurar la formación y el rol del profesor, alcanzar la ecología del aprendizaje, ${ }^{3}$ considerar todos los ámbitos educativos posibles, interactuar sobre los contenidos, ${ }^{4}$ encontrar una formación adaptada a las demandas, formar a ciudadanos y no solo a profesionales eficientes y evitar la ansiedad tecnológica. ${ }^{5}$

Todos estos retos de la educación del siglo XXI son grandes ideas desarrolladas por distintos especialistas en conferencias y artículos que podemos encontrar registrados en internet. Aquí nos interesa valorar aquellos en los que más directamente interviene la educación digital,

2 Se refiere a la idea de que las nuevas generaciones dominan las TIC y no necesitan formación en este terreno. Esta idea es falsa.

3 Sería conseguir que el aprendizaje redundase en las relaciones de los seres y con el medio en que viven.

4 El aprendizaje no reside en los contenidos sino en las interacciones que se producen en torno a ellos. Es decir, se deben poner en práctica los conocimientos y es aquí donde el alumno interviene agregando, modificando, criticando, reflexionando...; eso es interactuar y procesar un aprendizaje.

5 Los avances tecnológicos son rápidos y la educación tiene que usar los recursos del momento y aprovecharlos de forma rentable sin crear alumnos impacientes, inestables y ansiosos. 
que es el gran reto actual de la escuela debido al poder de la red como fuente de conocimiento y comunicación. La introducción de internet en las aulas es la parte instrumental más aparente de la metodología didáctica emergente.

\section{La educación digital}

Todos los supuestos retos de la educación del siglo XXI son consecuencia (aunque no exclusivamente) del desarrollo del mundo digital, que ha ampliado enormemente las fuentes de información y la manera de comunicarse. De los retos expuestos en el apartado anterior, el cuadro 2 recoge y explica aquellos intrínsecamente relacionados con la formación digital.

Cuadro 2 - Cómo influye la educación digital

\begin{tabular}{|l|l|}
\hline \multicolumn{2}{|c|}{ la educación digital } \\
\hline formar al ciudadano del siglo XXI & $\begin{array}{l}\text { para que sea competente con el } \\
\text { mundo digital }\end{array}$ \\
\hline extraer la inteligencia colectiva & $\begin{array}{l}\text { aprovechar las posibilidades } \\
\text { abiertas y múltiples de la sociedad } \\
\text { digital }\end{array}$ \\
\hline contenidos + pedagogía + & $\begin{array}{l}\text { la tecnología amplía las } \\
\text { posibilidades de la pedagogía } \\
\text { porque permite conocer mejor } \\
\text { tecnología }\end{array}$ \\
$\begin{array}{l}\text { y más y permite desarrollar el } \\
\text { aprendizaje significativo o por } \\
\text { descubrimiento del alumno } \\
\text { (el alumno como investigador } \\
\text { protagonista de su aprendizaje) }\end{array}$ \\
\hline
\end{tabular}




\section{la educación digital}

\begin{tabular}{|l|l|}
\hline $\begin{array}{l}\text { las TIC implican nuevos métodos } \\
\text { de evaluación }\end{array}$ & $\begin{array}{l}\text { no se evalúan solo contenidos sino } \\
\text { también la habilidad de adquirir y } \\
\text { poner en práctica esos contenidos }\end{array}$ \\
\hline $\begin{array}{l}\text { objetivo: desarrollo de } \\
\text { competencias }\end{array}$ & $\begin{array}{l}\text { las TIC permiten desarrollar el } \\
\text { trabajo autónomo, la capacidad } \\
\text { de investigación, el tratamiento } \\
\text { de la información, el trabajo } \\
\text { colaborativo, la comunicación... }\end{array}$ \\
\hline nuevo rol del profesor y su & $\begin{array}{l}\text { el profesor pasa a ser un guía } \\
\text { en el proceso de aprendizaje } \\
\text { y debe conocer perfectamente } \\
\text { formación }\end{array}$ \\
$\begin{array}{l}\text { los recursos que ayudarán al } \\
\text { alumno a conseguir su objetivo de } \\
\text { aprendizaje; por eso, debe tener } \\
\text { muchos conocimientos teóricos } \\
\text { y también prácticos, dominar la } \\
\text { fuentes de información y los flujos } \\
\text { y estrategias de búsquedas }\end{array}$ \\
\hline interacción sobre los contenidos & $\begin{array}{l}\text { el trabajo en red, con muchos } \\
\text { puntos de información y crítica, } \\
\text { permite al alumno pensar e } \\
\text { interactuar ante los problemas o } \\
\text { contenidos, pues debe, a partir de } \\
\text { varias fuentes, elaborar su propio } \\
\text { contenido en un ejercicio de } \\
\text { síntesis, abstracción, contraste... }\end{array}$ \\
\hline
\end{tabular}

Fuente: Rosa Ana Martín Vegas.

De este modo, se puede concluir (figura 1), que la educación digital resulta ser fundamental para el desarrollo de los principios del aprendizaje deseable. 
Figura 1 - presupuestos implicados en la educación digital

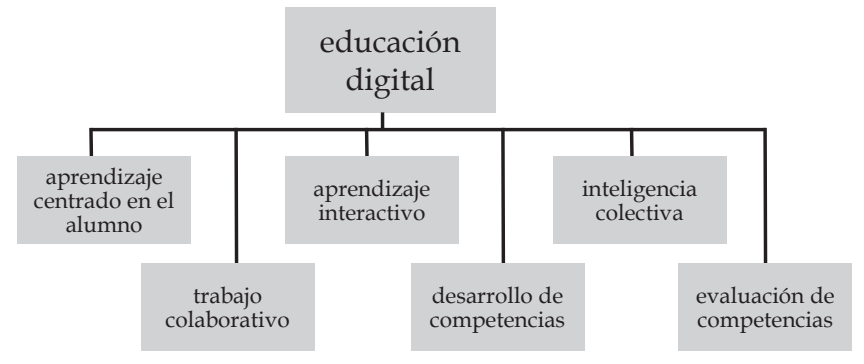

Fuente: Rosa Ana Martín Vegas.

La implantación de una educación digital ha supuesto muchos problemas, lo que indica que, pese a ser algo natural porque las nuevas tecnologías están totalmente instauradas en nuestra sociedad, la escuela es reticente. En principio se habló de la falta de apoyo y financiación por parte de las administraciones y de la falta de preparación de los docentes. Miller (2016) añade a la resistencia al cambio y a la formación del profesorado, las propias dificultades que implica el aprendizaje que exige una participación muy activa del alumno y el mismo aprendizaje personalizado. La mayeútica ${ }^{6}$ es complicada como método, pues exige inteligencia y habilidad por parte del profesor y también por parte del alumno, cuya disposición no siempre es favorable. Sin duda la metodología es tan compleja como enriquecedora.

Otra cuestión ligada a la educación digital ha sido la proliferación de cursos en línea. Pensando en la libertad

6 Método socrático de enseñanza que consiste en hacer descubrir los conocimientos al alumno a través de preguntas. 
que estos cursos dan al estudiante, muchas entidades y universidades han desarrollado cursos no presenciales. Pardo (2016) considera que la autonomía que ofrece el trabajar en línea da una flexibilidad muy agradecida por estudiantes y profesores. A su vez, el contacto más personal con el alumno parece una ventaja, pues se acercaría a la enseñanza más personalizada. Sin embargo, no es exactamente así porque las tutorías acercan las relaciones personales pero no personalizan el trabajo, pues los materiales sirven al gran grupo y las tareas y objetivos planteados son igual para todos. El contacto profesor-alumno en tutorías telemáticas puede ser bueno pero no mejor que el existente en una tutoría presencial. Corbalán (2001) habla de los problemas de la enseñanza en línea en Estados Unidos hace más de una década. Pues bien, los mismos problemas se mantienen hoy: mayor deserción en los cursos en línea que en los presenciales y dificultades a la hora de evaluar (exámenes tipo test en su mayoría, que evalúan más conocimientos que capacidades). A estas ideas, se debe añadir otra fundamental: los materiales de estos cursos son un calco a los libros de texto tradicionales; varía la conexión hipertextual que permiten los textos web y el diseño de opcionalidad de los ejercicios, que está limitado por los sistemas de corrección automática. Algo fundamental tiene que cambiar en la educación digital: el planteamiento metodológico y el uso de las fuentes de información, que no pueden exponerse como libros de textos, idénticos en formulación y metodología a los libros en papel pero en formato digital. 


\section{Libro de texto vs. trabajo por proyectos}

La difusión de materiales educativos digitales en estos últimos años no ha supuesto un gran cambio pedagógico respecto al uso de los libros de texto en papel como principal recurso para la enseñanza-aprendizaje en todas las materias. Los materiales digitales publicados son exactos a los libros de texto tradicionales, solo cambia el formato. Esta es la razón por la que no han supuesto más cambio en el planteamiento educativo que el puramente formal: en vez de leer en papel, se lee en pantalla y en vez de resolver los ejercicios en un cuaderno, se resuelven de forma digital, con poco texto escrito y mucho enlace o cliqueado. El gran cambio metodológico que no se ha llevado a cabo, al menos de manera extensiva, vendría de la mano de los llamados trabajos por proyectos.

Penalva (2016) señala el abandono del libro de texto como el paso esencial para conseguir un cambio en la educación. Considera el libro de texto clásico una herramienta obsoleta porque resume conocimientos aislados, sin contextualización y sin criterio de selección, pues no responden a las experiencias previas del alumno $\mathrm{ni}$ a sus motivaciones e intereses. Las limitaciones del libro de texto se deben a su formato, pues deben incluir conocimientos concretos según un programa educativo de orden ministerial. Por el contrario, el formato web, dadas las posibilidades de hipervincular textos, permite el acceso a conocimientos globales, de carácter más universal, pues la información puede ampliarse hasta donde se desee o necesite. Los materiales de internet son más plurales, pueden adaptarse mejor al contexto y al nivel 
de los alumnos y, sin duda, necesitan de la intervención activa del alumno para construir su aprendizaje. Nacen, de este modo, los trabajos por proyectos, que son planteamientos basados en temas de actualidad que deben desarrollarse buscando información en internet integrando conocimientos diversos y buscando un fin práctico que sea de interés para los alumnos. Estos proyectos didácticos están definidos en su planteamiento y guía de trabajo pero no en su desarrollo, pues cada estudiante, en función de sus posibilidades y sus intereses, moldeará su proyecto en función de unos fines. La selección de información es fundamental para el cauce que ha de seguir el proyecto, pero capacidades como la abstracción, la deducción y la inducción, así como la creatividad, desarrollan en cada caso proyectos personalizados distintos donde los alumnos son los auténticos protagonistas como participantes en la elaboración del trabajo y el procesamiento de la información. Es así como se produce el auténtico aprendizaje consciente. Esta metodología de trabajo que tiene como principal recurso internet, supone un verdadero cambio en el proceso educativo (ver figura 2). Y este cambio va más allá del uso de las TIC, porque no se trata de realizar actividades en formato digital, sino de procesar información para solucionar problemas o cuestiones de interés a partir de recursos digitales, que no son más que una fuente de información ingente por la cantidad de datos objetivos y subjetivos que pueden substraerse. 
Figura 2 - Aspectos metodológicos del trabajo por proyectos

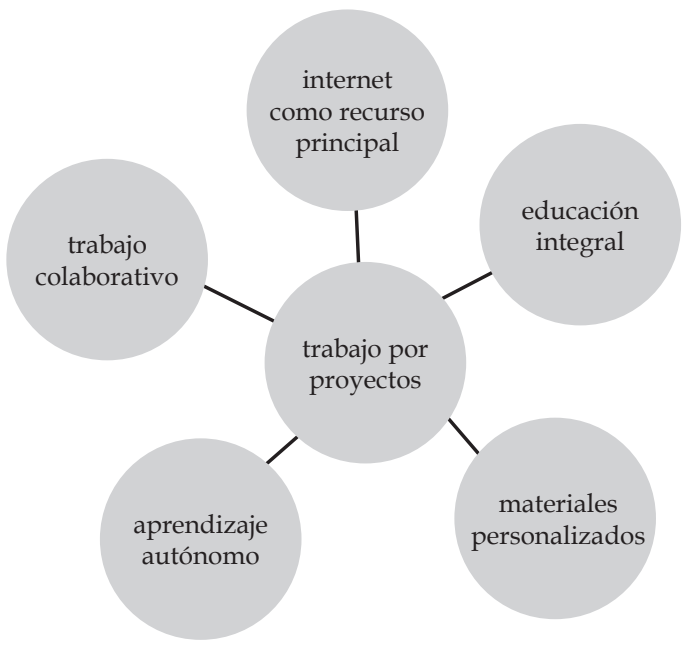

Fuente: Rosa Ana Martín Vegas.

La educación integral supone una transformación porque rompe con los conocimientos estancos, con la estructuración de asignaturas sin relación directa entre ellas; es una educación transversal. Por otra parte, relaciona aprendizaje conceptual, con procedimental y actitudinal, es decir, se relacionan conocimientos, con competencias y habilidades y con valores. Para elaborar este tipo de proyectos y poder trabajar en el aula con esta metodología, es imprescindible la labor del profesor, que necesita tener una buena formación en todos los sentidos, ser muy hábil en el proceso de comunicación y tener una buena disposición para trabajar mucho más de lo que supone la clase tradicional guiada por el libro de texto. Los proyectos exigen crear materiales de elaboración propia, pues la actualización de contenidos y la personalización adaptada 
al contexto requiere un esquema de trabajo previo muy estudiado y planificado. Este inmenso trabajo explica el éxito de los libros de texto, que facilitan al docente toda la labor educativa e incluso formativa, en muchos casos.

Los trabajos por proyectos tienen como principal fuente de información internet, la gran red de datos interconectados. Sin embargo, no todo está en internet. Los libros siguen siendo una fuente de información imprescindible; ${ }^{7}$ de este modo, las bibliotecas convencionales son centros de recursos fundamentales para la elaboración de los proyectos. ${ }^{8}$ Asimismo, todos los espacios sociales que puedan incorporarse al proyecto, así como todas las manifestaciones expresivas (escribir, hablar, grabar, actual, dibujar, cantar...) son parte de la idea de transversalidad y globalidad. ${ }^{9}$

$7 \quad$ El mismo libro de texto pasaría a ser un recurso más, no el único recurso, como se viene haciendo habitualmente. El rechazo principal al libro de texto viene dado porque fomenta un aprendizaje memorístico, exclusivamente basado en la transmisión de conocimientos y no en el desarrollo de competencias y destrezas.

8 En Martín Vegas (2014) se exponen las bases para el uso de la biblioteca escolar como un centro de investigación para el desarrollo de trabajo por proyectos.

9 En la cultura hispánica las salidas de los escolares del centro educativo son puntuales (excursiones esporádicas); sin embargo, en otras culturas como la germánica es más frecuente que los alumnos salgan del centro para ir a aprender matemáticas al mercado o ver insectos en el bosque cercano. La formación que implique, además del contacto con la vida real, servicios comunitarios (como puede ser ayudar a compañeros con discapacidades, hacer tareas de jardinería en el colegio, etc.) es mucho más funcional e interesante para los escolares. 
El trabajo por proyectos implica una mayor coordinación entre el profesorado y la comunidad educativa. Un proyecto de relativa difusión en España es LOVA (La Ópera como Vehículo de Aprendizaje, http:// proyectolova.es/): se ha llevado a cabo en escuelas de enseñanza primaria y secundaria y consiste en realizar un musical teatralizado con los niños cuya preparación requiere todo un curso escolar y la colaboración de todas las asignaturas. El interés de estos proyectos exige dedicación, ilusión y formación por parte del profesorado, que lleva el peso de la organización y del trabajo colaborativo.

Sanmartín (2015) considera que, junto a la desconexión del mundo real que presenta la educación actual, es la falta de calidad del profesorado su mayor problema. Se hace eco de los datos de una encuesta dada a conocer en la Cumbre Mundial de Educación de Qatar (WISE) donde se les pregunta a profesores, estudiantes, políticos y empresarios de 149 países. La formación inicial de los profesores debe reforzarse, pues no existe en la práctica universitaria una buena combinación entre formación conceptual y didáctica (MARTíN, 2015). Pero también la formación continua debe ser imprescindible en la carrera docente. Los cursos de formación, las jornadas para el contraste de prácticas educativas y otros encuentros con distintos formatos que permitan el intercambio profesional, serán muy motivadores para los profesores que, dispuestos a trabajar con sus alumnos, tengan el gusto por el conocimiento y la ampliación de campos metodológicos con el fin de descubrir el mundo a sus estudiantes. Los profesores no pueden dejar de aprender para enseñar. La formación de equipos de investigación didáctica en los colegios es muy interesante para la calidad educativa $y$, sin duda, 
solo se pueden llevar a cabo con colaboración y una buena gestión por parte de los directivos y de la administración que facilite esta labor y la incentive.

Otro problema de la educación actual es la evaluación. Sugata Mitra, profesor de la Universidad de Newcastle y ganador del TED Prize 2013, dice que "los exámenes ya no sirven, son una amenaza" (TORRES, 2016). Considera que hay que acabar con los programas académicos para situar internet en el centro de aprendizaje. Una metodología de trabajo por proyectos plantearía cambios porque no se evalúan solo conocimientos, como en los tradicionales exámenes, sino procesos de trabajo e investigación, que incluyen razonamiento, creatividad, maneras de hacer...

De esta manera, podemos concluir que los trabajos por proyectos responden a un aprendizaje mucho más consciente, válido a largo plazo en la vida de los alumnos $\mathrm{y}$ totalmente funcional y actualizado. Los requerimientos son muchos pero, principalmente, es necesario una buena formación y disposición profesional por parte del profesorado, que debe saber llevar a sus alumnos en ese proceso de aprendizaje extrayendo y desarrollando sus mejores cualidades y capacidades para prepararlos en la vida social y profesional. El cambio educativo requerido es metodológico y, en este sentido, sí se puede hablar de una revolución educativa que implique una "revolución de la lectura" y el desarrollo de la creatividad.

\section{La revolución de la lectura}

El filósofo Emilio Lledó, Premio Princesa de Asturias 2015 de Comunicación y Humanidades, habla de la necesidad de una "revolución de la lectura". La lectura libera la 
mente, permite aprender a pensar con autonomía, formar personas críticas y creativas. Vivimos una época en que se lee y se escribe mucho, pero en un formato que ha dado lugar a una forma muy distinta de lectura y de comunicación escrita. Posado (2015) señala en qué se ha convertido la lectura en línea:

Nuestros terminales táctiles nos abren libros, revistas y periódicos. Pero me temo que nos quedamos en la portada, lo inmediato, la foto, el vídeo o el titular sensacionalista. Nuestra mente está sufriendo un estrés de inmediatez, todo acontece 'on line' y caduca al poco tiempo. No hay pausa y demora para la reflexión. Así es muy difícil entender cabalmente qué sucede (POSADO, 2015).

Esta lectura rápida y por encima responde a las necesidades de búsqueda que permite el formato web, con distintas tipografías que resaltan ideas y con hipervículos de apertura opcional. Y en este sentido cumple una alta funcionalidad en la que también hay que formar a los estudiantes para evitar pérdidas de tiempo. Pero el término "revolución de la lectura" de Lledó no se refiere a la lectura somera exigida en la selección de información; se refiere a la lectura profunda, a la que lleva al verdadero aprendizaje, al que supone transformación, interiorización de contenidos y emociones y procesamiento (figura 3). 
Figura 3 - ¿Qué es el aprendizaje?

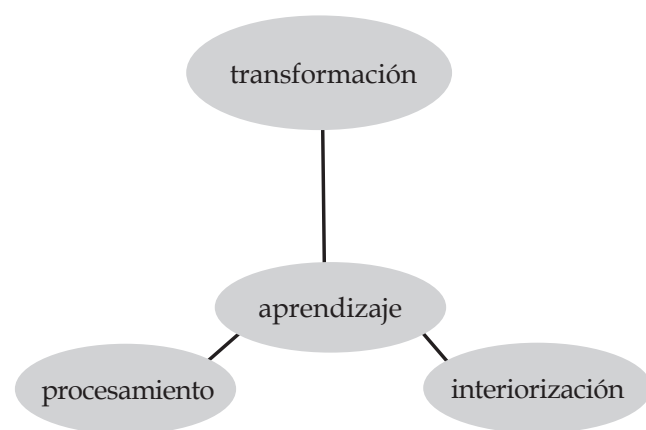

Fuente: Rosa Ana Martín Vegas.

Aprender a pensar es enseñar a aprender. $Y$ todo está inventado, la cuestión es ponerlo en práctica en las escuelas para acabar con el aprendizaje exclusivamente memorístico y conceptual que conduce a la desmotivación de tantos estudiantes por la falta de funcionalidad de los conocimientos aprendidos. El método para enseñar a aprender que ha de llevarse a cabo con la metodología de trabajo por proyectos (con todas las posibilidades posibles de trabajo que puedan imaginarse sobre cualquier tema) es la mayéutica socrática. Con este método, el maestro es un auténtico guía en el aprendizaje del alumno, pues a base de preguntas permite que los alumnos descubran los conocimientos gracias al acto de pensar. La labor del profesor es fundamental y para eso, como se ha comentado en el apartado anterior, ha de tener una excelente preparación y muy buenas aptitudes: debe saber conducir el proceso de aprendizaje mediante un buen planteamiento inicial de trabajo y buenos recursos que permitan la 
investigación del asunto. La técnica consiste en preguntar al alumno acerca del tema tratado y debatir las respuestas fundamentadas en conocimientos adquiridos en el desarrollo de la investigación. Poco a poco, los conocimientos se van ampliando y, gracias al saber, se encuentran soluciones a los problemas planteados. Este método permite desarrollar el pensamiento con todas sus implicaciones y prioriza el proceso de aprendizaje frente a los resultados. De esta manera, el alumno aprende realmente, porque es capaz de pensar por sí mismo gracias a su capacidad para realizar lecturas profundas, que relacionen lo que se lee con lo que se sabe del tema para extraer conclusiones propias que van más allá de la asimilación de unos contenidos transmitidos $\mathrm{y}$, a veces, no razonados.

\section{Conclusión: aprender a pensar para desarrollar la creatividad}

Hemos hablado de revolución de la educación y de revolución de la lectura, y todo esto, ¿para qué? El objetivo es desarrollar la creatividad, que permite desarrollar el talento de cada persona, que será necesario para la inserción social y profesional satisfactoria del estudiante en el futuro. En opinión de expertos en educación como Richard Gerver, la escuela actual mayoritaria condena la creatividad natural que tienen los niños. La escuela dirige tanto el aprendizaje ordenando a los niños qué deben aprender, cómo y en qué momento, que acaba con las dotes creativas que cualquier niño puede tener. La idea de Ken Robinson, orador y asesor en materia educativa de gobiernos e instituciones británicas y americanas, es que hay que poner a pensar a los niños para ayudarles a 
saber qué pueden hacer con lo que ya saben y con muchos más conocimientos que pueden adquirir. Esta es la idea expuesta en este artículo: hay que enseñar a pensar pensando y, para ello, es necesario un cambio metodológico que, con el uso de la tecnología digital, permita realizar la transformación necesaria para que el aprendizaje sea un procesamiento interiorizado por cada estudiante de forma que desarrolle todas sus capacidades intelectivas y creativas. Ayudarles a descubrir su talento es una tarea compleja pero posible.

\section{REFERENCIAS}

CASANOVA, O. Y. L. Bazarra. La escuela ya no es un lugar. Madrid: Arxic Formación, 2016.

CORBALÁN, R. Enseñanza digital en los Estados Unidos: ventajas y retos. En R. del Moral Aguilera, R. López Amate y A. Escobedo Rodríguez: Actas del XXXV Congreso Internacional de la Asociación Europea de Profesores de Español. Centro Virtual Cervantes: 73-77. Disponible en: <http:/ / cvc.cervantes.es/ensenanza/biblioteca_ele/ aepe/pdf/congreso_35/congreso_35_10.pdf>. 2001.

MARTÍN VEGAS, Rosa Ana Martin. La biblioteca escolar como centro de investigación. Mi biblioteca, n.38, p. 42-46. 2014.

MARTÍN VEGAS, Rosa Ana Martin. La Didáctica de la Lengua y la Literatura Españolas en el Máster en Formación del Profesorado de Educación Secundaria. Revisión y renovación. Revista española de pedagogía, n.261, p.365-379, mayo-ago. 2015. 
MILLER, F. Retos de la educación digital en 2016. Educación digital. Disponible en: < http://educacion. digital/retos-la-educacion-digital-2016/>

PARDO, I. La experiencia de la enseñanza en línea. Disponible en:<https://ismaelpardo.com/2016/09/19/ ensenaronline/>. 2016.

PENALVA, J. Así les va a los colegios e institutos que están acabando con el libro de texto. Xataka. Disponible en: <http://m.xataka.com/otros/asi-les-va-a-los-colegios-e-institutos-que-estan-acabando-con-el-libro-de-texto>. 2016.

PÉREZ-BARCO, M. J. Los veinte retos de la educación el siglo XXI. ABC.es. Disponible en: <http://www.abc. es/familia-educacion/20131211/abci-claves-educacion-201312101604.html>. 2013.

POSADO, J. La revolución de la lectura. La opinión. El correo de Zamora. Disponible em: <http://www.laopiniondezamora.es/opinion/2015/01/17/revolucion-lectura/816009.html>. 2015.

SANMARTÍN, O. R. (2015). Los cinco grandes problemas del profesorado español. El Mundo. Disponible en: <http:/ / www.elmundo.es/sociedad/2015/11/03/ 5637c9dc268e3e02488b456c.html>.

TORRES, Menárguez, A. (2016). Sugata Mitra: Los exámenes ya no sirven, son una amenaza. El País. Disponible en: <http:/ / economia.elpais.com/economia/2016/09/18/ actualidad/1474226496_636542.html>. 